\title{
Inoperable brain metastases from non-small cell lung cancer: what part does whole brain radiotherapy play in standard treatment?
}

\author{
Rachael Barton
}

Lung cancer is the second commonest cancer in the UK with an incidence in 2006 of 37100 , and is the commonest cause of death from cancer, causing more than 33000 deaths (22\% of cancer deaths) each year. ${ }^{1}$ More than three-quarters of lung cancers fall into the histological category of non-small cell lung cancer (NSCLC). Currently, 10\% of patients with NSCLC present with symptomatic brain metastases, and between a quarter and a third of those who initially survive radical treatment for stage III NSCLC will go on to develop brain metastases during their remaining life span. ${ }^{2}$ It is anticipated that over the next decade, an increasing proportion will develop brain metastases as adjuvant chemotherapy, concurrent chemoradiotherapy for locally advanced disease and chemotherapy for metastatic disease result in longer overall survival times.

For several decades, administration of steroids followed by whole brain radiotherapy (WBRT) has been standard treatment for inoperable multiple brain metastases of any primary cancer. The duration of survival for patients with a diagnosis of multiple brain metastases is strongly influenced by their performance status and also by the presence or absence of active extracranial disease. Data from three North American clinical trials which studied dose and fractionation for WBRT were used to divide patients with brain metastases from a variety of primary sites into three prognostic subgroups using recursive partitioning analysis (RPA). ${ }^{3}$ The group identified as having the best survival were aged less than 65 years, had a Karnofsky Performance Status score $\geqslant 70$ and had controlled primary disease with no metastases outside the brain. In

Correspondence to: Dr Rachael Barton, Princess Royal Hospital, Saltshouse Rd, Hull, East Yorkshire, HU8 9HE, UK; rachael.barton@hey.nhs.uk this group, termed RPA class I, median survival was 7.1 months. The group with the poorest survival (RPA class III) comprised those with a Karnofsky Performance Status score $<70$ and had a median survival of 2.3 months. The rest of the patients (RPA class II) did not fall into either of the other groups and had an intermediate median survival of 4.3 months. Patients with lung cancer accounted for $61 \%$ of the patients studied and almost all had NSCLC.

A prospective observational study has questioned the efficacy of WBRT for the treatment of brain metastases from solid malignancies. ${ }^{4}$ Of 75 patients treated with a standard WBRT regimen, only four could be shown to have an improved performance status at 1 month. At the same time point, $19 \%$ of those treated had experienced improvement in their presenting neurological symptom, $23 \%$ were stable and $55 \%$ were worse or had died. Patients fared little better when quality of life was considered: only 23 of 75 patients managed to complete quality of life forms at both baseline and 1 month; only 8 of these 23 patients had an improvement in their quality of life. The difficulties inherent in studying a group with such a poor prognosis have meant that reliable data on performance status and quality of life after WBRT are lacking.

Although survival times are short for patients with brain metastases from any primary cancer, data would suggest that patients with primary NSCLC have a worse prognosis than those with other primary malignancies and that their survival is at best measured in weeks. A study of 533 patients with brain metastases comparing two radiotherapy dose/ fractionation regimens was carried out by the Royal College of Radiologists, UK. ${ }^{5}$ The subgroup of patients with NSCLC (39\% of the total) had a median survival of just 69 days compared with 79 days for the group as a whole and 107 days for those with breast cancer. It is important to note that of the 533 patients randomised between the two fractionation regimens, only 352 (66\%) were available for assessment at the first time point, 4 weeks after treatment. The remainder had either died or were too ill to attend. This dismal prognosis is supported by prospectively collected data on 56 patients with brain metastases from primary NSCLC who were treated with WBRT in Newcastle upon Tyne, UK, between February 2001 and December 2005 (personal communication Dr Paula Mulvenna, 2006). The median survival of this population was 2.8 months even though $76 \%$ of the patients fell within RPA classes I and II.

It is clear that even after the current accepted "standard treatment" of steroids and WBRT, the survival of patients with brain metastases from NSCLC remains poor. It is therefore vital that their performance status and quality of life, which are often compromised by the effects of intracranial and extracranial disease, are not compromised further by the effects of treatment. ${ }^{6}$ Chow et al have documented deterioration in patient rated fatigue, drowsiness and appetite following WBRT in patients with brain metastases of various primary cancers. ${ }^{7}$ Clinical trials which have studied patients with brain metastases have largely addressed the question of radiotherapy dose and fractionation. There has been only one randomised controlled trial looking at the role of steroids with or without radiotherapy for brain metastases but this small trial pre-dates cerebral CT scanning and the results showed little benefit from addition of WBRT to supportive care and steroids. $^{8}$

The inclusion of WBRT as a part of standard treatment of brain metastases in NSCLC has been the subject of much discussion in recent years. The UK Consensus statement published in 2001 highlighted the need for clinical trials to address the palliative efficacy of WBRT.? A similar conclusion was reached by the National Cancer Institute of Canada workshop on symptom control in radiation oncology, published in 2003. ${ }^{10}$ The observed rapid deterioration in neurological function and performance status make it essential that such information is collected in the setting of a randomised clinical trial. This has led to the development of the QUARTZ trial (Quality of Life After Radiotherapy and Steroids). This trial is now open for recruitment in 
the UK and Australia and is being considered for approval in Canada.

QUARTZ is a Medical Research Council Phase III, randomised controlled trial designed to assess whether optimal supportive care alone is as effective as WBRT in combination with optimal supportive care in the treatment of patients with inoperable brain metastases from NSCLC. This is a pragmatic study in which the primary eligibility requirement is that the clinician and patient should be uncertain of the role of WBRT in their particular case. This should allow the majority of patients with inoperable brain metastases from NSCLC to be considered for inclusion in this trial and thus allow QUARTZ to produce robust evidence for or against the inclusion of WBRT in standard management. Patients with histologically or cytologically proven NSCLC and inoperable brain metastases are randomised between optimal supportive care with WBRT (standard treatment arm) and optimal supportive care alone (experimental arm). Patients who have previously received systemic treatment for their lung cancer are eligible. In the context of the QUARTZ trial, optimal supportive care is defined as the use of dexamethasone, titrated down to the lowest dose required to control symptoms, and: specialist nursing support; open access to follow-up in a specialist clinic; and access to a specialist palliative care multidisciplinary team. The primary end point of the QUARTZ trial is patient assessed quality adjusted time (OALY). Secondary end points are overall survival, Karnofsky Performance Status and neurological symptoms.

\section{SUMMARY}

Brain metastases from NSCLC are, sadly, all too common. They have a devastating effect on quality of life and functional ability and median survival of patients with unresectable disease is between 2 and 3 months. It is not clear whether whole brain radiotherapy adds anything to the quality or length of survival of such patients and a randomised clinical trial has just begun which is designed to address this issue. It is essential that the QUARTZ trial recruits as many eligible patients as possible if the results are to be meaningful. Patients with inoperable brain metastases from NSCLC should be discussed at a lung cancer multidisciplinary meeting. This provides an opportunity to debate the likely benefit of WBRT in each individual case and if there is uncertainty then entry into QUARTZ should be considered.

Competing interests: None.

Rachael Barton is a member of the QUARTZ Trial Management Group
Thorax 2008;63:1-2. doi:10.1136/thx.2007.086215

\section{REFERENCES}

\section{Cancer Research Campaign.}

www.cancerresearchuk.org laccessed 7 November 2007).

2. Barnholtz-Sloan JS, Sloan AE, Davis FG, et al Incidence proportions of brain metastases in patients diagnosed (1973 to 2001) in the Metropolitan Detroit Cancer Surveillance System. J Clin Oncol 2004:22:2865-72.

3. Gaspar L, Scott C, Rotman M, et al. Recursive partitioning analysis (RPA) of prognostic factors in three Radiation Therapy Oncology Group (RTOG) brain metastases trials. Int J Radiat Oncol Biol Phys 1997;37:745-51.

4. Bezjak A, Adam J, Barton R, et al. Symptom response after palliative radiotherapy for patients with brain metastases. Eur J Cancer 2002;38:487-96.

5. Priestman TJ, Dunn J, Brada M, et al. Final results of the RCR trial comparing two different radiotherapy schedules in the treatment of cerebral metastases. Clin Oncol 1996;8:308-15.

6. Gerrard GE, Prestwich RJ, Edwards A, et al. Investigating the palliative efficacy of whole-brain radiotherapy for patients with multiple-brain metastases and poor prognostic features Clin Oncol 2003;15:422-8.

7. Chow E, Davis L, Holden L, et al. Prospective assessment of patient-rated symptoms following whole brain radiotherapy for brain metastases. J Pain Symptom Manage 2005;30:18-23.

8. Horton J, Baxter DH, Olson KB, et al. The management of metastases to the brain by irradiation and corticosteroids. Am J Roentgenol Radium Ther Nucl Med 1971:111:334-36.

9. Hoskin PJ, Brada M. Consensus Statement: radiotherapy for brain metastases. Clin Oncol 2001;13:91-4.

10. Tsao MN, Sultanem K, Chiu D, et al. Supportive care management of brain metastases: what is known and what we need to know. Clin Oncol 2003;15:429-34.

\section{Aspirin sensitivity and eicosanoids}

\section{Sophie Farooque, Tak H Lee}

Aspirin sensitive respiratory disease (ASRD) was first described in 1922 by the French physician Widal. ${ }^{1}$ It is characterised by asthma, chronic rhinosinusitis and nasal polyps on a background of aspirin sensitivity. The condition is a distinct, often aggressive, clinical syndrome, and it is rare in childhood with a

King's College London, MRC and Asthma UK Centre in Allergic Mechanisms of Asthma, Guy's Hospital, London, UK

Correspondence to: Professor Tak H Lee, Department of Asthma, Allergy and Respiratory Science, 5th Floor, Thomas Guy House, Guy's Hospital, London SE1 9RT, UK; tak.lee@kcl.ac.uk peak age of onset in the early 30 s. $^{2}$ Rhinorrhoea and nasal congestion are typically the first symptoms with asthma usually manifesting 1-5 years after the onset of rhinitis. ${ }^{3}$ Once the disease is established, ingestion of aspirin induces the release of critical mediators that provoke an acute exacerbation of rhinosinusitis and asthma. It is estimated that 5$10 \%$ of all patients with asthma are aspirin sensitive. ${ }^{4}$ Often poorly responsive to treatment, patients with aspirin sensitivity are over-represented in the severe asthma group and $50 \%$ are steroid dependent..$^{5}$

The aetiology of ASRD is complex, but most investigators are agreed that the reaction to aspirin is not mediated by allergic mechanisms. Most evidence points towards an abnormality of arachidonic acid (AA) metabolism. AA is a substrate for both the production of leucotrienes (via the 5-lipoxygenase (5LO) pathway) and prostanoids (via the cyclooxygenase (COX) pathway).

ASRD is characterised by excessive cysteinyl leucotriene (CysLT) production both in the steady state and for several hours after aspirin challenge. ${ }^{6}$ Urinary leucotriene E4 ( $\left.\mathrm{LTE}_{4}\right)$ levels, as a measure of total body production of CysLTs, are a mean sixfold higher in patients with ASRD, increasing fourfold higher still after aspirin challenge. ${ }^{7}$ To date, the question of whether ASRD is associated with a fundamental predetermined abnormality in the production of CysLT ${ }^{8}$ or whether it is an expression of particularly severe disease remains unresolved. ${ }^{9} 10$ Furthermore, while the mucosal cellular infiltrate resembles that of asthma and rhinitis generally, there is even greater 